\title{
Clinical appearance of vascular compromise after filler injection
}

\author{
Dong-Hak Jung, MD, PhD, Sang Min Hyun, MD, PhD, Kit Liang Gan, MBBS, MRCSI, MS \\ Shimmian Rhinoplasty Clinic, Seoul, Rep. of Korea
}

\begin{abstract}
Background: Although filler injections are established as safe and effective for facial rejuvenation, they have been associated with adverse outcomes. Vascular compromise is one of the most severe complications arising from facial filler injections, either as inadvertent intra-arterial embolization or vascular compression, which leads to localized skin necrosis or vision loss.

Objective: This study aimed to estimate the clinical course and causes of vascular compromise after filler injections by conducting a retrospective analysis.

Methods: We retrospectively analyzed 30 patients (28 females; mean age, 35.1 years; age range, $24-45$ years) with vascular compromise in the nose after receiving filler injections between June 1, 2010 and June 30, 2015. All the patients began treatment of vascular compromise within 5 days after receiving filler injection. We retrospectively analyzed the patterns of filler necrosis by using medical photographs and records.

Results: The pattern of vascular compromise followed the blood vessel distribution. As the vessels are connected to each other, they are difficult to classify clearly. However, they can be divided into lesions on the nasal bridge, nasal tip, nasal ala; lesions including more than 2 subunits; and lesions accompanied by surrounding structures such as the lips. In addition, the pattern and outcome of the lesions differed according to the cause, either arterial embolization or vascular compression.

Conclusion: Vascular compromise resulting from filler injection can have serious consequences. As necrosis causes serious problems for both the patient and the physician, the possibility of necrosis must be recognized and prevented in advance, and immediate treatment should be performed when symptoms develop.
\end{abstract}

Keywords: arterial embolization; filler injection; vascular compression; vascular compromise

\section{Introduction}

Filler injections are established as safe and effective for facial rejuvenation with immediate aesthetic effects. The substances contained in fillers include hyaluronic acid (HA) products, collagen, paraffin, and liquid silicone. Fillers also can be classified as temporary, semi-permanent, and permanent fillers, according to by the duration of their effects [1]. In the past, permanent fillers were widely used, but in recent years, the HA filler, which is a kind of absorbable filler, is the most popularly used. This specific popularity may be caused by the potential reversibility of HA fillers with hyaluronidase.

Although filler injections are touted to be safe, they have been associated with adverse outcomes. These complications range from bruising, erythema, migration, and nodule formation to more serious sequelae such as vascular compromise. Vascular compromise is one of the most devastating complications arising from facial filler injections, either as inadvertent intra- 
arterial embolization or vascular compression, which leads to localized skin necrosis or vision loss [2-4].

Vascular compromise usually appears in 2 forms, namely arterial embolization and vascular compression. This study aimed to estimate the clinical course and cause of vascular compromise after filler injection by conducting a retrospective analysis.

\section{Materials and methods}

We retrospectively evaluated 30 patients (28 females; mean

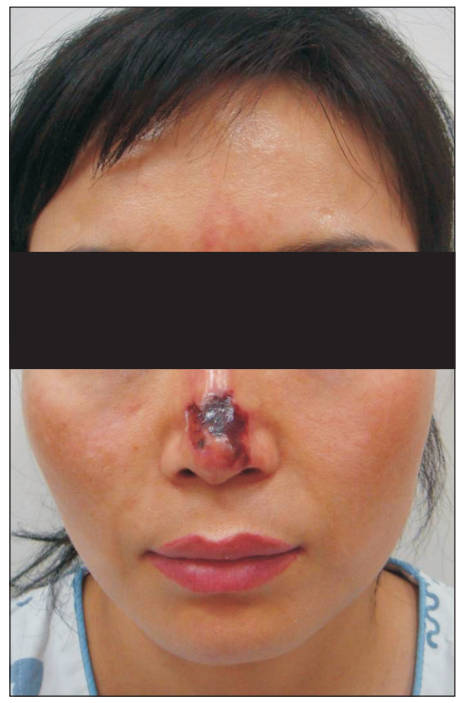

Fig. 1. A lesion of vascular compromise on the nasal bridge is shown.

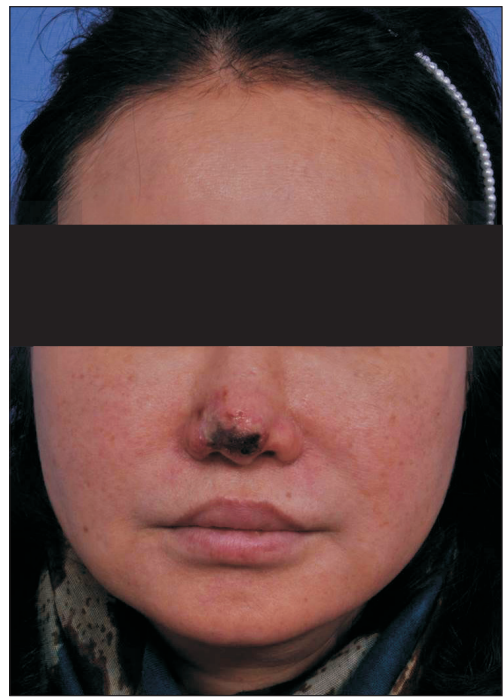

Fig. 2. The image depicts a lesion of vascular compromise on the nasal tip. age 35.1 years; age range, 24-45 years) who visited our clinic to undergo treatment for vascular compromise in the nose after filler injection, between June 1, 2010 and June 30, 2015. All the patients began treatment of vascular compromise within 5 days after filler injection. We retrospectively analyzed the pattern of filler necrosis by using medical photographs and records.

Each patient was given an opportunity to review the manuscript and consented to its publication.

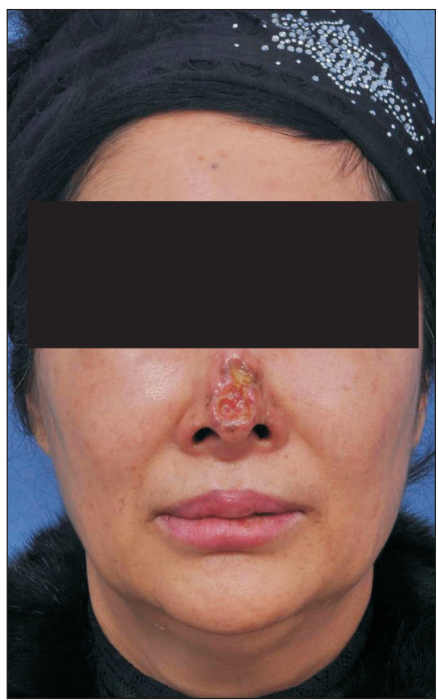

Fig. 3. A lesion of vascular compromise on the nasal tip and bridge is demonstrated.

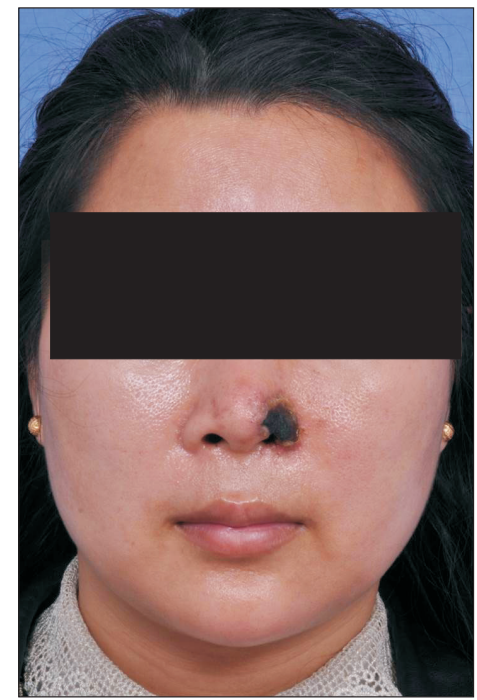

Fig. 4. An example of a lesion of vascular compromise on the nasal ala is shown. 


\section{Results}

The pattern of vascular compromise followed the blood vessel distribution. As the vessels are connected to each other, they are difficult to classify clearly. However, they can be divided into lesions on the nasal bridge (6.7\%), nasal tip (6.7\%), nasal ala (20.0\%), and glabella (6.7\%); lesions including more than 2 subunits (43.3\%); broad lesions (10.0\%); and lesions accompanied by surrounding structures such as the lips (10.0\%). In addition, the pattern and outcome of the lesions differed according to the cause, either arterial embolization or vascular compression.

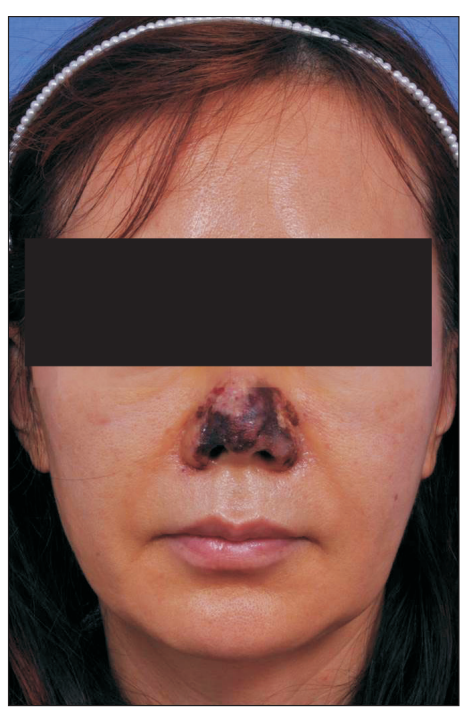

Fig. 5. A lesion of vascular compromise on the nasal tip and ala is shown.

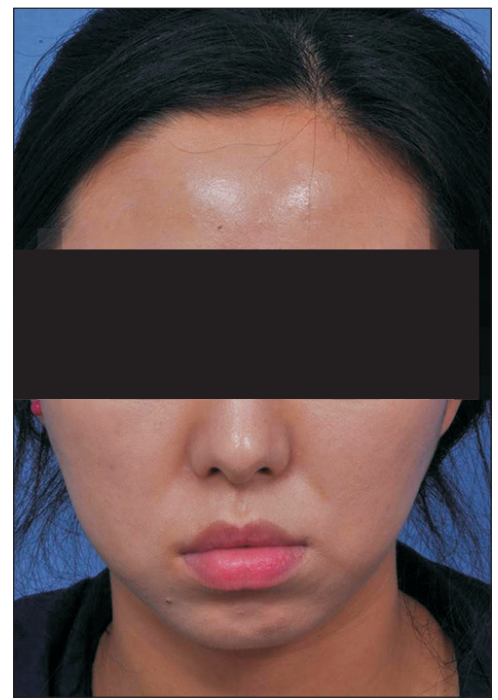

Fig. 6. The image shows a lesion of vascular compromise on the glabella.

\section{Distribution pattern of nasal vascular compromise}

\section{Nasal bridge}

The lesion on the nasal bridge can be assumed to be associated with the dorsal nasal artery (Fig. 1).

\section{Nasal tip}

The lesion can be assumed to be associated with the columellar and lateral nasal arteries (Fig. 2).

\section{Nasal tip and bridge}

Obstruction of the columellar branch, dorsal nasal artery, and lateral nasal artery could be associated with nasal tip and bridge lesions (Fig. 3).

\section{Nasal ala}

Obstruction of the lateral nasal artery may cause nasal alar necrosis. The lesion may occur on both sides of the ala but is uncommon. Unilateral lesions of alar necrosis are one of the most common patterns of vascular compromise (Fig. 4).

\section{Nasal tip and ala}

The lesion on the nasal tip and ala is thought to be related to obstruction of the columellar branch and lateral nasal artery. This lesion is the most common form of vascular compromise after filler injection in the nasolabial fold, as the lateral nasal artery is a major source of blood supply to the nasal tip and ala (Fig. 5).

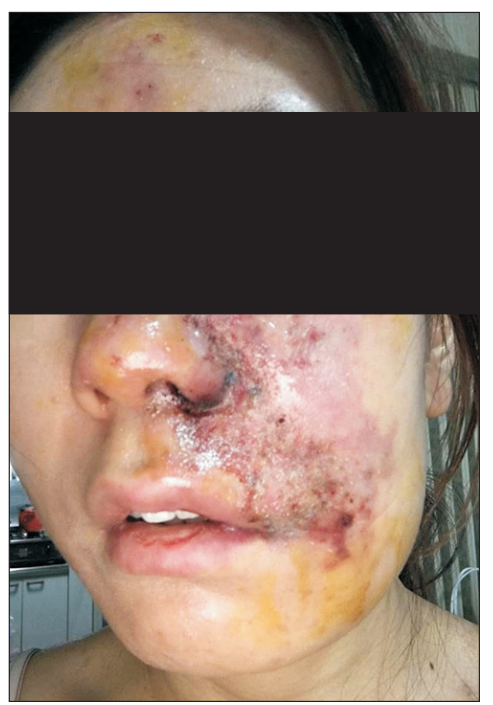

Fig. 7. A broad lesion of vascular compromise is demonstrated. 


\section{Glabella}

Glabellar lesions can occur alone but are usually accompanied by lesions of the nasal dorsum (Fig. 6).

\section{Broad lesion}

The lesion can be assumed to be associated with the angular artery (Fig. 7).

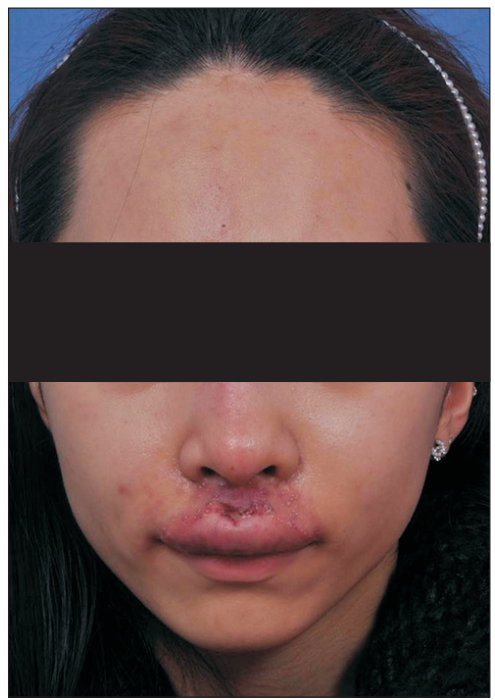

Fig. 8. A lesion can occur in combination with other structures.

\section{Combined with other structures}

The facial artery is presumed to be involved before branching to the angular artery (Fig. 8).

\section{Comparison of arterial embolization and vascular compression}

\section{Arterial embolization}

Within 5 days after filler injection, eschar is generated on the lesion. If a partial lesion has developed, the prognosis is better than that of broad lesions but leaves a severe scar and sequelae on the nose. In the case of arterial embolization, necrosis progresses rapidly in a broad area, consequently requiring reconstructive surgery (Fig. 9).

\section{Vascular compression}

Lesions caused by vascular compression may cause bullae and blisters on the skin, and may be accompanied by small pustules. Erythematous lesions may present throughout the skin, with some turning red with dark red portions, but necrosis does not progress rapidly. Widespread lesions may retain some sequelae despite early treatment, but if the lesion is not widespread, it may recover without any sequelae through early treatment (Fig. 10).
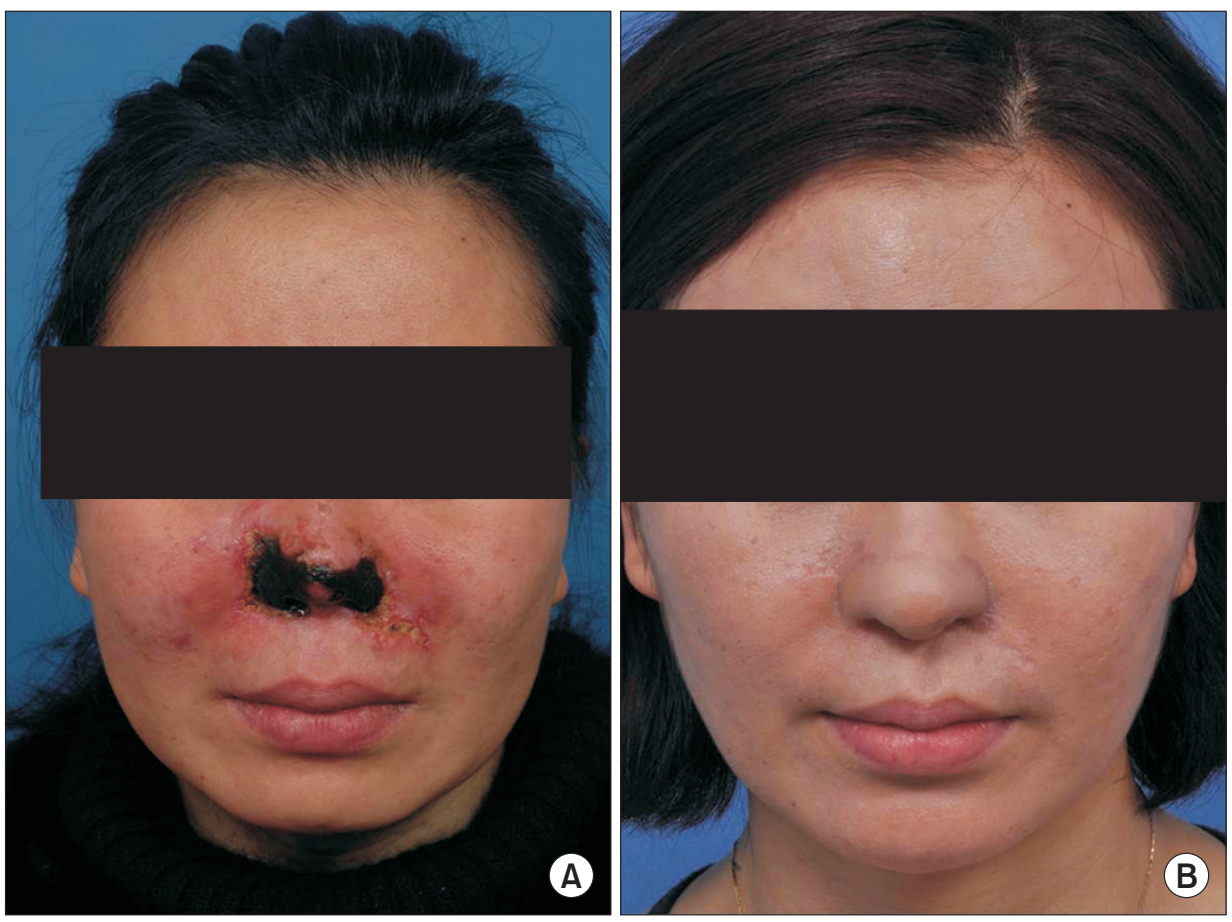

Fig. 9. (A) Arterial embolization leaves a severe scar and sequelae. (B) Consequently, reconstructive surgery is necessary. 

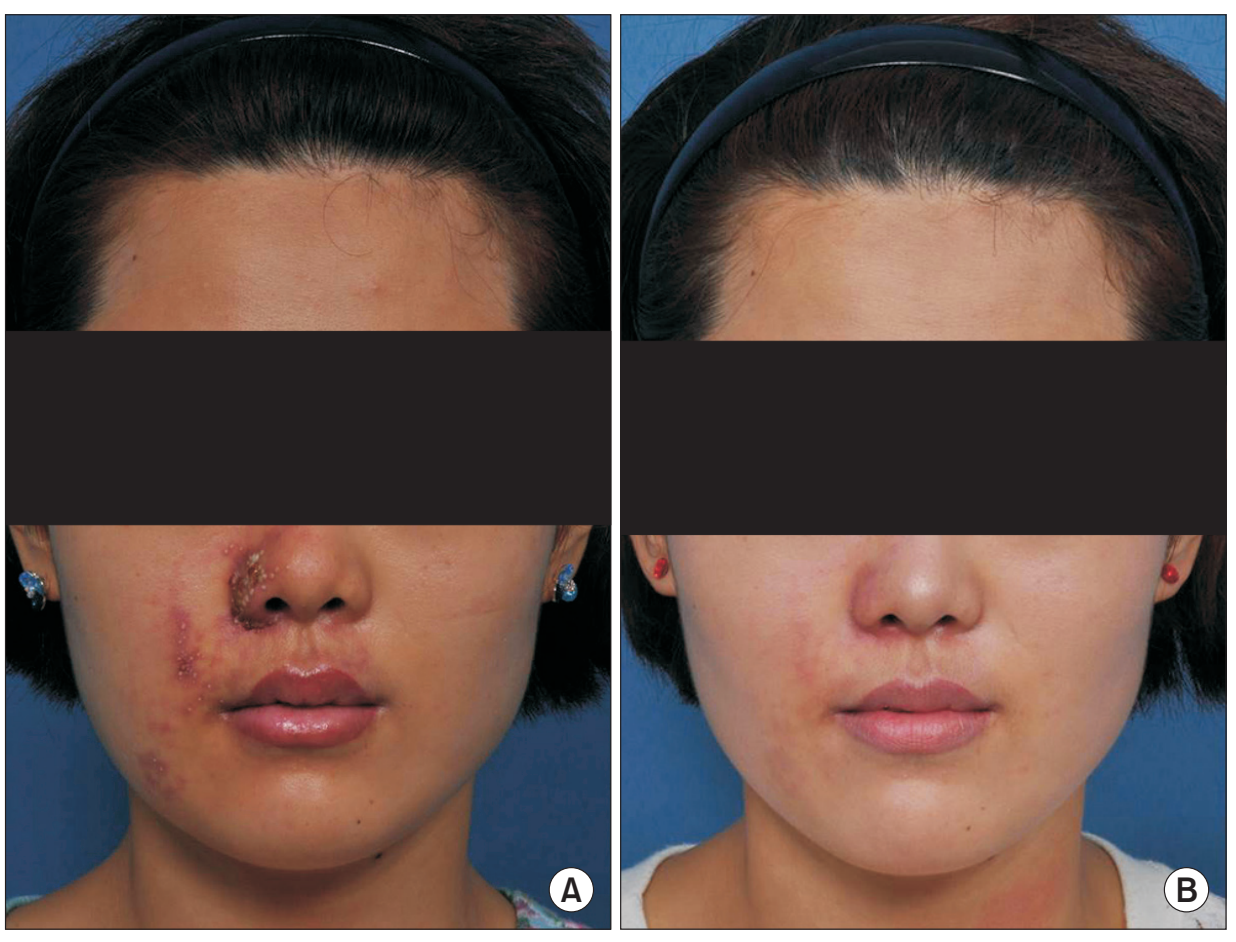

Fig. 10. (A) A lesion caused by vascular compression appears as bullae and blisters, and may accompany small pustules. (B) Lesions of vascular compression usually do not progress to necrosis but leave sequelae in the case of widespread lesions.

\section{Discussion}

Filler rejuvenation is commonly performed because of its safety and short recovery time after the procedure. However, owing to the occurrence of lethal side effects such as vascular compromise, continuous studies on filler procedures and side effects are needed. The sources of blood supply to the nose consist of the columellar branch, dorsal nasal artery, and lateral nasal artery. However, sometimes variation in blood supply is observed. The dorsal nasal artery would be predominant with a small or absent lateral nasal artery, while the lateral nasal artery dominates in some cases without the dorsal nasal artery [5]. The variation of blood supply suggests that collateral blood supply could be absent when the artery is obstructed. The most common site for filler injections is the face, including the nasolabial fold and nose. Even if the injection is performed on the lip or cheek, vascular compromise on the nose may occur because of anastomotic blood supply.

We divided the causes of vascular compromise into arterial embolization and vascular compression. Venous occlusion is also possible during cosmetic injection. Instead of immediate pain and blanching, this presents with venous mottling termed livedo. Venous occlusion was successfully treated with heat, massage, hyaluronidase, and prednisolone therapies without any sequelae [2]. Vascular compression lesions cause blisters and erythematous lesions on the skin. As the skin color turns dark red, abrasion or erosion occurs. The occurrence of eschar is rare, and some scar may be left if the lesion is widespread. However, in the case of arterial embolization, a vesicle or blister occurs in part after blanching, but localized eschar or erosion occurs within 5 days. Arterial embolization is more commonly direct anterograde, with occlusion of an artery causing ischemia distal to the injection point. However, retrograde flow of filler against the arterial pressure to an arterial bifurcation followed by anterograde flow causes vascular occlusion in the retina and thus blindness through the ophthalmic and central retinal arteries. Vascular compromise could occur owing to arterial embolization solely. However, in some cases, vascular compromise is caused by both vascular compression and embolization. Recent studies have suggested the clinical presentation and time of occurrence of ischemia as the blanching, livedo reticularis (marbling), blue-gray, blister, demarcation, and ulceration phases [6].

Prevention of vascular compromise is more important than the treatment of filler necrosis. Awareness of several features of vascular compromise is important for its prevention. Choosing safe materials first and familiarization with the anatomical knowledge of the injection site are important, as well as to check for vascular injury by regurgitation, if possible, during the injection. Use of a catheter or blunt needle with a hole on the side of the syringe rather than a sharp needle is suggested. After the injection, the patient should be checked for blanching, pain, 
or heating sensation. If the pain or heat persists after the patient returns home and the skin turns to pale, the patient should be instructed to contact the clinic without delay.

Once the progression of vascular compromise is confirmed, intensive treatment should begin immediately. Injection of a minimum of 200 to $300 \mathrm{U}$ of hyaluronidase (spread over the entire area of impending necrosis) is essential. Injection of hyaluronidase should be repeated daily for a minimum of 2 days until signs of permanent necrosis or reestablished blood flow appears. Doses up to $1,500 \mathrm{U}$ were suggested if needed $[3,7]$. In addition, hyperbaric oxygen and intravenous prostaglandins should be given 2 times a day for more than 7 days. Warm massage, oral aspirin, prophylactic antibiotics, and topical steroids would be helpful.

If severe scarring or deformation is caused by vascular compromise, reconstructive surgery may be necessary. In general, reconstructive surgery is recommended to be performed after stabilization of surrounding tissues for several months after necrosis and after the lesion has become smaller. However, in some cases, reconstructive surgery is performed within a few weeks after necrosis.

Depending on the size or location of the deformed area, the necrotic lesion may be excised and reconstructed using a local flap such as a rhomboid or bilobed flap. Reconstructive surgery is basically performed in 3 stages, and one or more additional surgeries may be required. The type of flap and method of reconstruction should be determined in consideration of various factors.

In conclusion, vascular compromise resulting from filler injection can have serious consequences. The pattern of vascular compromise follows the vasculature of the nose. The pattern also depends on different mechanisms of vascular compression and arterial embolization. As necrosis causes serious problems for both the patient and the physician, the possibility of necrosis must be recognized and prevented in advance and immediate treatment should be performed when symptoms develop.

\section{Conflicts of interest}

The authors have nothing to disclose.

\section{References}

1. Moon HJ. Injection rhinoplasty using filler. Facial Plast Surg Clin North Am 2018;26:323-30.

2. Woodward J, Khan T, Martin J. Facial filler complications. Facial Plast Surg Clin North Am 2015;23:447-58.

3. Signorini M, Liew S, Sundaram H, De Boulle KL, Goodman GJ, Monheit G, et al.; Global Aesthetics Consensus Group. Global aesthetics consensus: avoidance and management of complications from hyaluronic acid fillers-evidence- and opinion-based review and consensus recommendations. Plast Reconstr Surg 2016;137:961e-71e.

4. Inoue K, Sato K, Matsumoto D, Gonda K, Yoshimura K. Arterial embolization and skin necrosis of the nasal ala following injection of dermal fillers. Plast Reconstr Surg 2008;121:127e$8 \mathrm{e}$.

5. Jung DH, Kim HJ, Koh KS, Oh CS, Kim KS, Yoon JH, et al. Arterial supply of the nasal tip in Asians. Laryngoscope 2000;110:308-11.

6. Snozzi P, van Loghem JAJ. Complication management following rejuvenation procedures with hyaluronic acid fillers-an algorithm-based approach. Plast Reconstr Surg Glob Open 2018;6:e2061.

7. Hong JY, Seok J, Ahn GR, Jang YJ, Li K, Kim BJ. Impending skin necrosis after dermal filler injection: a "golden time" for first-aid intervention. Dermatol Ther 2017;30:e12440. 